\title{
Long-term photometry of blazars at Abastumani Observatory
}

\author{
Omar M. Kurtanidze, Maria G. Nikolashvili, Givi N. Kimeridze, \\ Lorand A. Sigua, Bidzina Z. Kapanadze and Roman Z. Ivanidze
}

Abastumani Astrophysical Observatory, 383792 Abastumani, Georgia

\begin{abstract}
We give a brief summary of the ongoing Abastumani Active Galactic Nuclei Monitoring Program started in the May 1997. More than 110000 frames are obtained during more than 1300 nights of observations for about 50 target objects, among them gamma-ray, X-ray and optical blazars. All observations were done in the BVRI bands using ST-6 CCD based photometer attached to the Newtonian focus of $70-\mathrm{cm}$ meniscus telescope.
\end{abstract}

Keywords. BL Lacertae objects - variability

\section{Introduction}

Among active galactic nuclei (AGNs), blazars are objects most dominated by nonthermal continuum emission, which extends from radio to gamma-rays, and whose properties are best explained by emitting plasma in relativistic motion towards the observer, closely aligned with the line of sight (Urry 1995). One of the distinguishing characteristics of the blazars which include BL Lacertae type objects, high polarization quasars (HPQ) and optical violently variable (OVV) quasars is that their flux densities are highly variable at all wavelength from radio to gamma-rays. The optical multiband monitoring along with other ones gives unique clues into the size and structure of the radiating region.

Variability timescales have been derived for many blazars from monitoring programs which attain a time resolution of days and to years. Unfortunately, existing multiwavelength data are not adequate yet to permit definite conclusions to be drawn about the nature of blazars due to the optical coverage in the previous campaigns have been much to sparse. We started systematic multi-wavelength optical monitoring of blazars at Abastumani Observatory in the may of 1997. In the late of October 1997 we joined the Whole Earth Blazar Telescope (WEBT, http://www.to.astro.it/blazars/webt). The aim of the program is to study short-term and long-term variability of blazars and their correlations with that in radio, X-ray and gamma-ray bands.

\section{Observation and data reduction}

Abastumani Observatory is located in the South-Western part of Georgia at a latitude of $41.8 \mathrm{grad}$ and a longitude of $42.8 \mathrm{grad}$ on the top of the Mt. Kanobili at $1700 \mathrm{~m}$ above mean sea level. The weather and seeing are very good in Abastumani (150 nights per year, one third with seeing less than 1 arcsec). The mean values of the night sky brightness are $\mathrm{B}=22.0 \mathrm{mag}, \mathrm{V}=21.2 \mathrm{mag}, \mathrm{R}=20.6 \mathrm{mag}$, and $\mathrm{I}=19.8 \mathrm{mag}$. Blazar Monitoring Program at Abastumani Observatory was started in the May 1997 and is carried out with Petlier cooled CCD Imaging Camera attached to the Newtonian focus of the 70-cm meniscus telescope (1:3). The pointing accuracy of the meniscus telescope is one-two arcminutes and it is good enough to locate target object inside of the full frame field of view $14.9 \times 10.7$ square arcminute. 
The ST-6 Imaging Camera uses the TC241 chip (375x242, 23x27 sq. micron) with a maximum quantum efficiency 0.7 at $675 \mathrm{~nm}$. All observations were performed using combined filters of glasses which match the standard B, V (Johson) and Rc, Ic (Cousins) bands well. Reference sequences in the blazar fields are calibrated using the Landolt's equatorial standard stars (Landolt 1992). In photometric nights at least one equatorial field is observed with different exposures. Because of the scaling of CCD and resolution $\mathrm{f}$ the meniscus telescope are equal to $2.3 \times 2.7 \mathrm{sq}$. arcsec per pixel and 1.5 arcsec respectively, the images are under-sampled, therefore to improve sampling it is needed to defocus frames slightly. Unfortunately, the high dark current limits the exposure time to 900 sec. The images are reduced using Daophot-II (Stetson 1987). To eliminate the effects of seeing induced spurious IDV and IHV (Cellone 2000) the apertures are taken to include the whole host galaxy. The highest differential photometrical accuracy reached in R-band is $0.005 \mathrm{mag}(\mathrm{rms})$ magnitude at $\mathrm{m}(\mathrm{R})=14.00 \mathrm{mag}$ during $180 \mathrm{sec}$.

\section{Results and conclusions}

List of target objects was compiled using two catalogues of AGNs (Padovani 1996, Perlman 1996). In the period from May 1997 to September 2005 (about 1450 observing nights) more than 130000 frames were obtained. In the table the list of the target objects along with the number of nights observed and frames obtained in every of the BVRI bands in excess of one hundred fifty in $\mathrm{R}$ band are given. Last column shows the number of frames obtained to study the intra-day and intra-hour variability. Among most frequent observed objects are BL Lacertae, S5 0716+714, AO 0235+164 and Mkr 421.

So far we took part in many international multi-wavelength campaigns conducted during outburst and post-outburst era of BL Lacertae, S5 0716+714, AO 0235+164, Mkr 421, 3C 279, 4C 29.45, OJ 287, S4 0954+650 and ON 231. The main part of the results are already published (Ostorero 2006; Osterman 2006; Villata 2006; Krawczynski 2004; Jorstad 2004; Raiteri 2005; Bottcher 2005). Most objects under study show light variations over one magnitude in the optical band. Largest one was observed for AO $0235+164$ and equals to 4.0 mag in R-band. A few faint variable stars (B 16.5 mag) with amplitude $0.3-0.4 \mathrm{mag}$ and periods $3-5$ hours were also identified.

\section{Acknowledgements}

O.M.K. gratefully acknowledge financial support and the kind collaboration with Dr. G. M. Richter without which this Programme would never have been conducted. We thank European Astronomical Society for donation of the ST-6 CCD camera.

\section{References}

Bottcher, M. et al. 2005, ApJ, 631, 169

Celone, S. A. et al. 2000, AJ, 121, 90

Jorstad, S. et al. 2004, AIPC CS, 714, 202

Krawczynski, H. et al. 2004, ApJ, 601, 151

Landolt, A. U. 1992, AJ, 104, 340

Osterman, A. M. et al. 2006, A\&A, 132, 873

Ostorero, L. et al. 2006, A\&A, 451, 797

Padovani, P., Giommi, P. et al. 1996, MNRAS, 277, 1477

Perlman, E. et al. 1996, A\&A, 424, 497

Stetson, P. B. 1987, PASP, 99, 19

Villata, M. et al. 2004, A\&A, 453, 817

Raiteri, C. M., Villata, M. et al. 2005, A\&A, 438, 39

Urry, C. M., Padovani, P. et al. 1995, PASP, 107, 803 